\title{
MODELOS ESTOCÁSTICOS APLICADOS À AVALIAÇÃO DINÂMICA DA PAISAGEM FLORESTAL DE REMANESCENTES DE MATA ATLÂNTICA NO MUNICÍPIO DE CAXIAS DO SUL-RS ${ }^{1}$
}

\author{
Gisele Cemin ${ }^{2}$ e Jorge Ricardo Ducati ${ }^{3}$
}

\begin{abstract}
RESUMO - A conversão de áreas naturais para a produção agrícola e exploração imobiliária levou a uma redução considerável das áreas florestais do bioma Mata Atlântica no último século. Dados oficiais apontam redução de $71 \%$ das florestas contidas nesse bioma, sendo um indicativo preocupante, uma vez que é reconhecida mundialmente como o quinto dos 34 hot spots do planeta, abrigando alta biodiversidade e elevado grau de endemismo. Nesse contexto, o objetivo deste estudo foi avaliar a evolução da fragmentação florestal do bioma Mata Atlântica no Município de Caxias do Sul, RS, calibrando um modelo dinâmico espacial desse processo e simulando um cenário futuro para o ano 2021. Para atingir o objetivo proposto, foi utilizada uma série temporal de imagens do satélite Landsat 5 referentes aos anos 1985, 2004 e 2011, dados do relevo e também informações pedológicas. Os resultados indicaram aumento da cobertura florestal nativa de 1985 a 2011 (incremento de 36\%) e também do cenário simulado (2021) de $20 \%$ de áreas florestais. Esse aumento da cobertura florestal na área avaliada está possivelmente associado ao êxodo rural, maior rigor na aplicação da legislação ambiental e fiscalização rígida por parte do órgão ambiental. Essa informação apresenta relevância na tomada de decisão no que tange à gestão e fiscalização dos recursos florestais.
\end{abstract}

Palavras-chave: Sensoriamento remoto; Autômatos celulares; Fragmentação.

\section{STOCHASTIC MODELS APPLIED TO DYNAMIC EVALUATION OF FOREST LANDSCAPE OF REMAININGS FROM ATLANTIC FOREST IN THE CITY OF CAXIAS DO SUL-RS}

\begin{abstract}
The conversion of natural areas for agricultural production and the real estate exploitation has led to a considerable reduction of forest areas in the Atlantic Forest in the last century. Official data indicate a reduction of $71 \%$ in this biome's forests, what is a worrying indicator, since it is worldwide recognized as the fifth of the 34 hot spots of the planet and with high biodiversity so as high degree of endemism. In this context, the aim of this study was to evaluate the changes in the forest fragmentation of the Atlantic forest in the city of Caxias do Sul-RS, calibrating a spatial dynamic model for this process and simulating a scenario for the year of 2021. To achieve the proposed goal it was used a time series of Landsat 5 satellite images from the years of 1985, 2004 and 2011, with topography and soil information data. The results indicated an increase of native forest from 1985 to 2011 (increase of 36\%) and to the 2021 simulated scenario (20\% offorest areas). This increase in forest cover in the evaluated area is possibly associated with the rural exodus, more rigorous enforcement of environmental laws and strict enforcement by the environmental agency. This presents relevant information for decision making in relation to the management and monitoring of forest resources.
\end{abstract}

Keywords: Remote Sensing; Cellular automata; Fragmentation.

\footnotetext{
${ }^{1}$ Recebido em 29.10.2014 aceito para publicação em 11.09.2015.

${ }^{2}$ Universidade de Caxias do Sul, Instituto de Saneamento Ambiental, Caxias do Sul, RS - Brasil. E-mail: <gicemin@yahoo.com.br>.

${ }^{3}$ Universidade Federal do Rio Grande do Sul, Instituto de Física, Departamento de Astronomia, Porto Alegre, RS - Brasil. E-mail: <ducati@if.ufrgs.br>.
} 


\section{INTRODUÇÃO}

O histórico de degradação e desmatamento dos hábitats florestais em nível mundial é muito antigo, datando de mais de 20.000 anos e persistindo até os dias atuais (FAO, 2007). No caso do Brasil, o histórico de desmatamento e de degradação das florestas começou provavelmente há mais de 13 mil anos (DEAN, 1996) com os povos antigos (caçador-coletor), que se utilizavam do fogo para suas atividades. Esse processo de ocupação e de degradação se intensificou com a chegada dos povos europeus há mais de 500 anos (FONSECA, 1985). Dados divulgados pelo Ministério do Meio Ambiente (2007) e pelo Instituto Nacional de Pesquisas Espacial (2014) apontaram perda de floresta tropical no território brasileiro na ordem de 18,85\% na Amazônia e 71\% na Mata Atlântica. Esse dado é preocupante, uma vez que o Brasil é o país com maior biodiversidade do planeta, abrigando entre 10 e $20 \%$ das espécies e $30 \%$ das florestas tropicais do mundo (LEWINSOHN, 2005).

A Mata Atlântica, alvo deste estudo, é considerada uma das mais ameaçadas, já que perdeu e continua perdendo áreas naturais. É reconhecida mundialmente como o quinto dos 34 hot spots do planeta (MYERS et al., 2000; MITTERMEIER et al., 2004), reserva da Biosfera pela Unesco e Patrimônio Nacional pela Constituição Federal de 1988, sendo esses indicativos relevantes da sua importância para a manutenção da biodiversidade e deve receber prioridade na conservação biológica. Quando os primeiros europeus se estabeleceram na costa brasileira, a Mata Atlântica ocupava mais de 1,3 milhão de $\mathrm{km}^{2}$. Hoje, aproximadamente $27 \%$ da sua área original ainda permanece com cobertura florestal nativa. Além da perda de hábitat e da biodiversidade, a Mata Atlântica atingiu estágio avançado de fragmentação, em que menos de $7 \%$ das florestas remanescentes estão localizadas em fragmentos com mais de 100 hectares (MYERS et al., 2000; MESQUITA et al., 2011). Esses fragmentos se encontram, em grande parte, isolados uns dos outros e são compostos por florestas secundárias em estágios iniciais e médios de regeneração (METZGER et al., 2009). Além disso, apresentam em seus domínios cerca de $70 \%$ da população nacional (MMA, 2002), o que torna críticas tentativas de preservação desse bioma.

Nesse aspecto, os dados derivados de sensoriamento remoto constituem ferramenta amplamente utilizada para identificar e quantificar a distribuição das áreas florestais desde a escala global até a local (MYERS, 1988; ACHARD et al., 2002). Essa ferramenta, além de facilitar a observação de um objeto, sem a necessidade de contato físico, apresenta a vantagem de permitir a análise de processos dinâmicos devido à coleta repetitiva de informações de um mesmo ponto da superfície terrestre. Outra aplicação inovadora do sensoriamento remoto é na alimentação de modelos dinâmicos que simulam cenários futuros do uso e cobertura do solo. Os mapas produzidos em uma série histórica podem servir de subsídio para determinar o processo de mudança e alimentar o modelo dinâmico, que tem como premissa conhecer as taxas de transição entre as classes observadas de uso e cobertura do solo.

Um dos modelos de simulação mais empregados em estudos da dinâmica da paisagem é o modelo matemático estocástico-probabilístico. Por meio desse modelo é possível simular as áreas futuras mais suscetíveis a novas alterações de uso, tendo como base uma matriz de transição definida a partir de mudanças de uso do solo em datas passadas e a vinculação dessas mudanças a certos critérios definidos por pesos de evidências (GOODCHILD, 1992). Entre os modelos de simulação de cenários futuros estocásticoprobabilístico, as cadeias de Markov acopladas a algoritmos que consideram as células vizinhas no espaço, como os autômatos celulares, constituem uma das melhores técnicas atualmente disponíveis para responder às necessidades e interesses das investigações acerca da dinâmica do uso do solo urbano e regional (ALMEIDA et al., 2007). Esse modelo possui simplicidade operacional, matemática e a facilidade com que pode utilizar dados provenientes do Sensoriamento Remoto. Além disso, é de fácil implementação em sistemas de informações geográficas e, ainda, não necessita de grande quantidade de dados do passado para prever o futuro (PEDROSA; CÂMARA, 2003). O processo básico de Markov, segundo Hobbs (1983), pode ser expresso pela notação matricial apresentada a seguir:

$$
\Pi(\mathrm{t}+1)=\mathrm{P}^{\mathrm{n}} \cdot \Pi(\mathrm{t})
$$

em que é um vetor coluna, com n elementos, representando a condição do sistema em um tempo t particular; П $(\mathrm{T}+1)$ é um vetor de ocupação nos $n$ Estados após o intervalo de tempo $\mathrm{t}+1$ e é a matriz de probabilidade de transição. 
A matriz de Markov modela as mudanças entre classes de uso e cobertura do solo de uma maneira multidirecional, ou seja, parcelas de uma classe podem teoricamente mudar de uma categoria mutuamente exclusiva para outra em um tempo qualquer. Portanto, três categorias de uso geram seis possibilidades diferentes de mudança, como disposto na seguinte matriz (SOARESFILHO, 1998):

$$
\mathrm{P}=\left[\begin{array}{lll}
\mathrm{P}_{11} & \mathrm{P}_{12} & \mathrm{P}_{13} \\
\mathrm{P}_{21} & \mathrm{P}_{22} & \mathrm{P}_{23} \\
\mathrm{P}_{31} & \mathrm{P}_{32} & \mathrm{P}_{33}
\end{array}\right]
$$

As probabilidades de transição são usualmente derivadas de amostras relativas a certo instante de tempo. Cadeias de Markov de primeira ordem assumem que o estado futuro do sistema depende apenas do seu estado presente e das possibilidades de transição, sendo independente da trajetória que o levou àquele estado (estados em um tempo t-1). Esse modelo não ignora o passado, mas assume que toda a informação do passado está concentrada no estado do sistema. Dessa forma, as interações são instantâneas, sendo irrelevante o tempo de permanência das variáveis em cada estado(SOARES-FILHO, 1998). Outra característica das cadeias de Markov é que as probabilidades de transição não mudam com o tempo, o que o caracteriza como processo estacionário.

No Brasil, pesquisas de maior relevância no meio científico que tratam da modelagem de fenômenos relacionados com as mudanças de uso do solo estão concentradas no bioma Amazônico, onde se destacam os trabalhos de Soares-Filho et al. (2004), SoaresFilho et al. (2006), Soares-Filho et al. (2007), Aguiar (2007), Flamenco-Sandoval et al. (2007), Maeda et al. (2011), Lima et al. (2013) e Piontekowski et al. (2013).

Nesse contexto, o objetivo deste trabalho foi calibrar um modelo dinâmico espacial baseado em autômatos celulares da fragmentação florestal do bioma Mata Atlântica no Município de Caxias do Sul, RS, entre os anos 1985 e 2011, simulando um cenário futuro para 2021. Os dados gerados poderão ser utilizados como ferramenta balizadora para a proposição de políticas públicas que visam à consonância entre o desenvolvimento socioeconômico e a preservação ambiental.

\section{MATERIAL E MÉTODOS}

\section{1 Área de estudo}

O Município de Caxias do Sul está localizado na região Nordeste do Estado do Rio Grande do Sul (entre as coordenadas Latitude Sul 28 $45^{\prime}, 2^{\circ} 25^{\prime}$ e Longitude Oeste $50^{\circ} 45^{\prime}, 51^{\circ} 20^{\prime}$ ), abrangendo uma área de aproximadamente $1.587 \mathrm{~km}^{2}$.

Está inserido na sua totalidade no bioma Mata Atlântica, o qual é constituído por um conjunto de formações vegetais com características diferenciadas, particularidades ecológicas e associações de espécies próprias. Observa-se a presença de três regiões fitoecológicas pertencentes a esse bioma, como se seguem: Floresta Ombrófila Mista-Mata com Araucária, Floresta Estacional Decidual e Estepe Gramíneo Lenhosa com Mata de Galeria (campos de altitude).

A Floresta Estacional Decidual está localizada, em sua maior parte, nos vales encaixados do rio TaquariAntas e Caí. A estrutura desta floresta é representada por dois estratos arbóreos distintos: um emergente, aberto e decíduo, com altura variando entre 25 e 30 $\mathrm{m}$, com destaque para o angico (Parapiptadenia rigida), a grápia (Apuleia leiocarpa) e a canafístula (Peltophorum dubium) e outro, dominado e contínuo, de altura não superior a $20 \mathrm{~m}$, formando, principalmente, por espécies perenifólias, além de um estrato de arvoretas, em que se observa a presença de espécies como Actinostemon, Soroceae e Trichilia. As palmáceas são representadas pela espécie Arecastrum romanzoffianun (jerivá), presente em todas as regiões florestais dos Sul do Brasil (TEIXEIRA; NETO, 1986).

A Floresta Ombrófila Mista ocupa os locais com altitude superior a $500 \mathrm{~m}$ acima do nível do mar, predominantemente sobre rochas de basaltos e rochas efusivas ácidas. O principal elemento dessa floresta é a Araucaria angustifolia (pinheiro-brasileiro), espécie endêmica do bioma, que, por sua relevante importância comercial, movimentou a economia de parte do Sul do Brasil nas décadas de 1960 e 1970, sendo posteriormente as áreas com araucárias desmatadas para fins agrícolas, especialmente para o plantio de soja e trigo e mais recentemente utilizadas para a implantação da monocultura de Pinus spp. (BEHLING; PILLAR, 2007).

A região de ocorrência da formação fitoecológica conhecida como estepe gramíneo-lenhosa com mata de galeria desenvolve-se em altitudes superiores a 800

Revista Árvore, Viçosa-MG, v.39, n.6, p.1019-1030, 2015

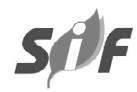




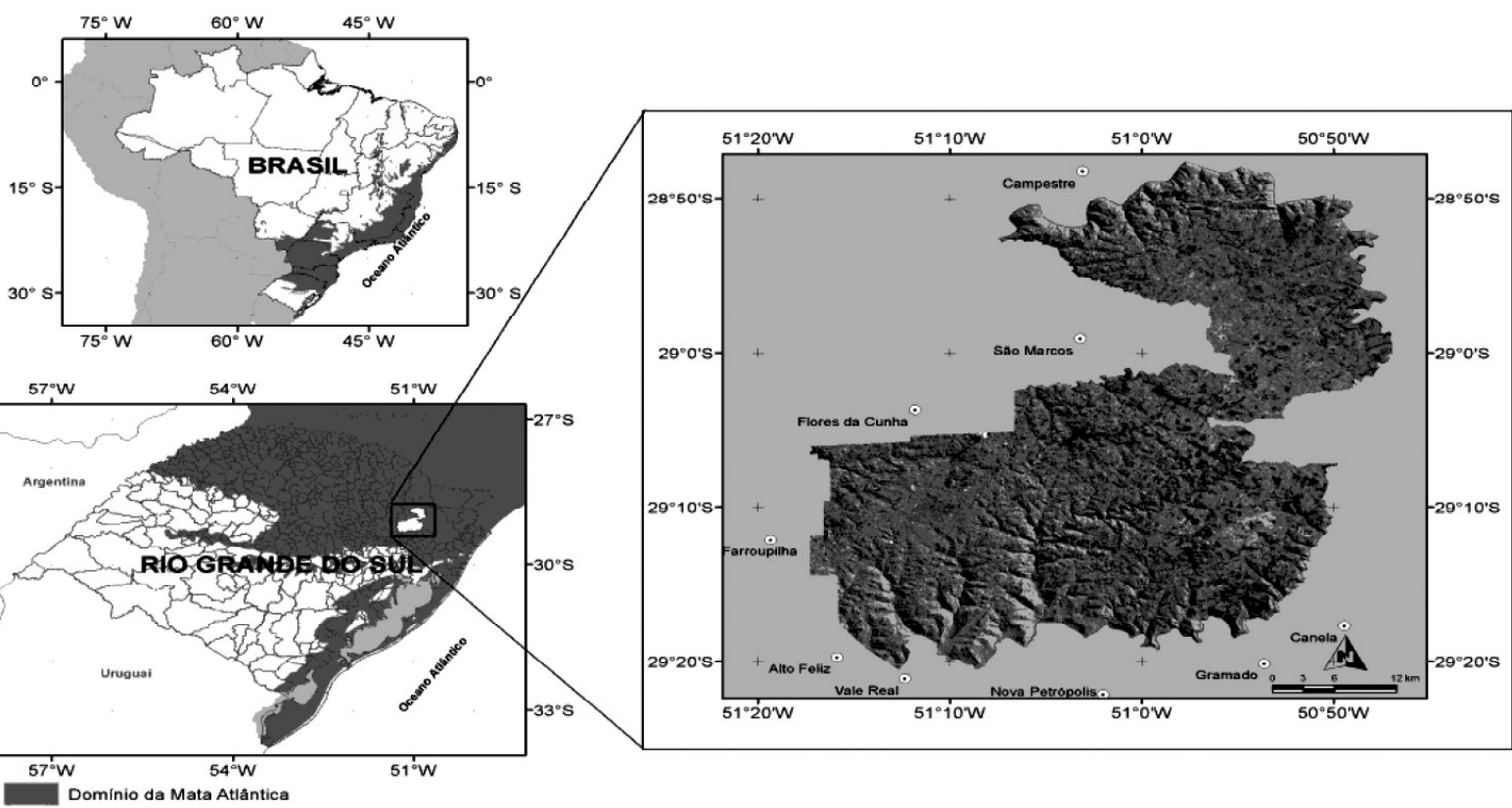

Figura 1 - Localização da área de estudo.

Figure 1-Location of the study area.

m acima do nível do mar, predominando na região Nordeste do município, sobre áreas de relevo ondulado como em áreas de relevo fortemente ondulado, na sua maior parte sobre solos rasos como Cambissolos e Latossolos, derivados de rochas efusivas ácidas e básicas (TEIXEIRA; NETO, 1986).

\subsection{Dados de uso e cobertura do solo}

A evolução temporal da paisagem para a calibração do modelo dinâmico espacial foi acompanhada a partir de um conjunto de imagens do sensor TM do satélite Landsat 5, com $30 \mathrm{~m}$ de resolução espacial. A investigação foi realizada nos anos de 1985 (07/12/ 1985), 2004 (08/10/2004) e 2011 (28/10/2011).

Os procedimentos metodológicos relacionados com processamento das imagens de satélite incluíram o georreferenciamento, a classificação e a quantificação das diferentes classes de uso e cobertura do solo presentes na paisagem. O georreferenciamento foi executado na imagem de 2011, utilizando pontos de controle coletados no mosaico de imagens GeoCOVER (GLCF, 2008). O erro médio quadrático (RMS) do georreferenciamento foi controlado para que se obtivesse um valor melhor que 1 pixel, ou seja, inferior a $30 \mathrm{~m}$. A imagem de satélite de 1985 e 2004 foram registradas utilizando a imagem georreferenciada de 2011 como referência.

Seguiu-se para a classificação das imagens de satélite de forma supervisionada utilizando o algoritmo de Máxima Verossimilhança Gaussiana. Foram elencadas cinco classes de uso e cobertura do solo para o Município de Caxias do Sul, a saber: floresta nativa, campo nativo, agrossilvipastoril, área urbana e lâmina d’água.

\subsection{Modelagem dinâmica espacial}

Para a estruturação de um modelo dinâmico espacial, com vistas à geração de um cenário futuro da paisagem do Município de Caxias do Sul, com ênfase na fragmentação da Mata Atlântica, foi utilizado o software DINAMINA (Landscape Dynamic System). Em linhas gerais, a modelagem dinâmica espacial foi dividida em cinco etapas, como seguem: i) Geração dos mapas de uso e cobertura do solo dos anos de 1985, 2004 e 2011; ii) Elaboração das variáveis estáticas: hipsometria, clinografia e pedologia; iii) Calibração e validação do modelo dinâmico para as mudanças observadas na paisagem no período de 1985 a 2004; iv) Com os dados parametrizados, calibrados e validados (primeira validação) das mudanças da paisagem de 1985 a 2004, projetou-se o cenário futuro para 2011, com o objetivo de verificar 
a concordância do dado simulado para o ano 2011 , com a imagem real classificada de 2011 (segunda validação); v) A partir da segunda validação do modelo projetou o cenário futuro para o ano 2021, utilizando os parâmetros calibrados e validados da etapa anterior para o período de 1985 a 2004.

As variáveis estáticas do relevo utilizadas no modelo dinâmico foram provenientes de dados cartográficos extraídos da base cartográfica digital do Rio Grande do Sul(HASENACK, 2010), na escala 1:50.000. A variável estática referente à pedologia foi obtida com base em informações bibliográficas e o mapeamento, realizado por Flores et al. (2007). Foram identificadas sete classes pedológicas na área de estudo, apresentadas em ordem decrescente de ocorrência: Neossolo litólico, Cambissolos húmicos, Cambissolos háplicos, Argissolo acinzentado, Nitossolo bruno, Gleissolo melânico e Chernossolo háplico. A variável referente à distância dos fragmentos florestais foi gerada pelo próprio software DINAMICA a partir do mapa de uso e cobertura do solo de entrada (mapa de 1985) e recalculada para cada ano modelado.

No processo de calibração do modelo, foram geradas as matrizes de transição anual (matriz unitária) e global (matriz periódica), as quais foram calculadas para um período de tempo considerado de 19 anos (1985 a 2004). Nesse procedimento, obtiveram-se as taxas de mudanças de uma classe de uso e cobertura do solo para outra classe. A matriz de transição global apresenta a taxa de transição de todo o período de tempo analisado, enquanto a matriz de transição anual mostra taxa de transição decomposta ano a ano.

Para o cálculo dos pesos de evidência, foram utilizados como dados de entrada os mapas de uso e cobertura do solo de 1985 e 2004 , as das variáveis estáticas combinadas em um único arquivo e os dados referentes às melhores faixas de distância (ou classe) de cada variável. Como saída, são calculados os pesos de evidências, representados pelos $\mathbf{W}+$ (peso que favorece uma transição na paisagem em determinada faixa de distância e em determinada variável) e pelo W- (peso que desfavorece uma transição na paisagem em determinada faixa de distância e em determinada variável).

A última etapa para a implementação do modelo dinâmico foi o ajuste das funções de transição, que podem ser por Expander (expansão ou retração de fragmentos preexistentes de dada classe de uso do solo) ou Patcher (nucleação - geração de novos fragmentos de dada classe). Essas funções foram geradas para cada ano a ser simulado, a partir dos pesos de evidências de cada variável, simulando quantitativa e espacialmente as transições na paisagem no período avaliado. A definição do percentual de Expander e Patcher foi concebida de forma heurística pelo modelador, com base no seu conhecimento do processo a ser modelado.

A primeira fase de validação dos resultados da paisagem simulada foi realizada mediante comparação heurística dos resultados observados e simulados. A segunda fase foi comparando os resultados do mapa de uso simulado com o de uso observado de forma matemática, analisando os resultados dentro de um contexto de vizinhança. Mesmo que os mapas não correspondem exatamente pixel-a-pixel, ainda assim podem apresentar padrões espaciais similares e, da mesma forma, correspondência espacial dentro de certa vizinhança de pixels. Para essa avaliação baseada em vizinhança, foram utilizados de Ajuste por Múltiplas Resoluções (Goodness of Fit - F) (CONSTANZA, 1989) e de Medida de Similaridade Fuzzy Modificado (S) adaptado de Hagen (2003). Uma vez que a validação apontou boa medida de similaridade $\mathrm{F}$ e $\mathrm{S}$ entre a simulação para o ano 2004 e o mapa real de uso e cobertura do mesmo ano, alterou-se a entrada do modelo pelo mapa real de uso e cobertura do solo no referido ano, com o objetivo de validar o modelo com a imagem real de 2011. Validado o modelo para o ano 2011, alterou-se o mapa de entrada para o uso e cobertura do solo real de 2011 e projetou-se um cenário de 10 anos correspondentes ao ano 2021, sendo todos os parâmetros utilizados validados para 2004.

\section{RESULTADOS}

Atendendo ao objetivo e à abordagem metodológica proposta, são apresentados os resultados referentes às áreas florestais do Município de Caxias do Sul, RS, nos anos 1985, 2004 e 2011, bem como o prognóstico para 2021, segundo a modelagem dinâmica espacial.

\subsection{Dados das áreas florestais}

A Figura 2 traz as informações de uso e cobertura do solo do Município de Caxias do Sul, nos anos 1985, 2004 e 2011 , respectivamente.

Revista Árvore, Viçosa-MG, v.39, n.6, p.1019-1030, 2015 


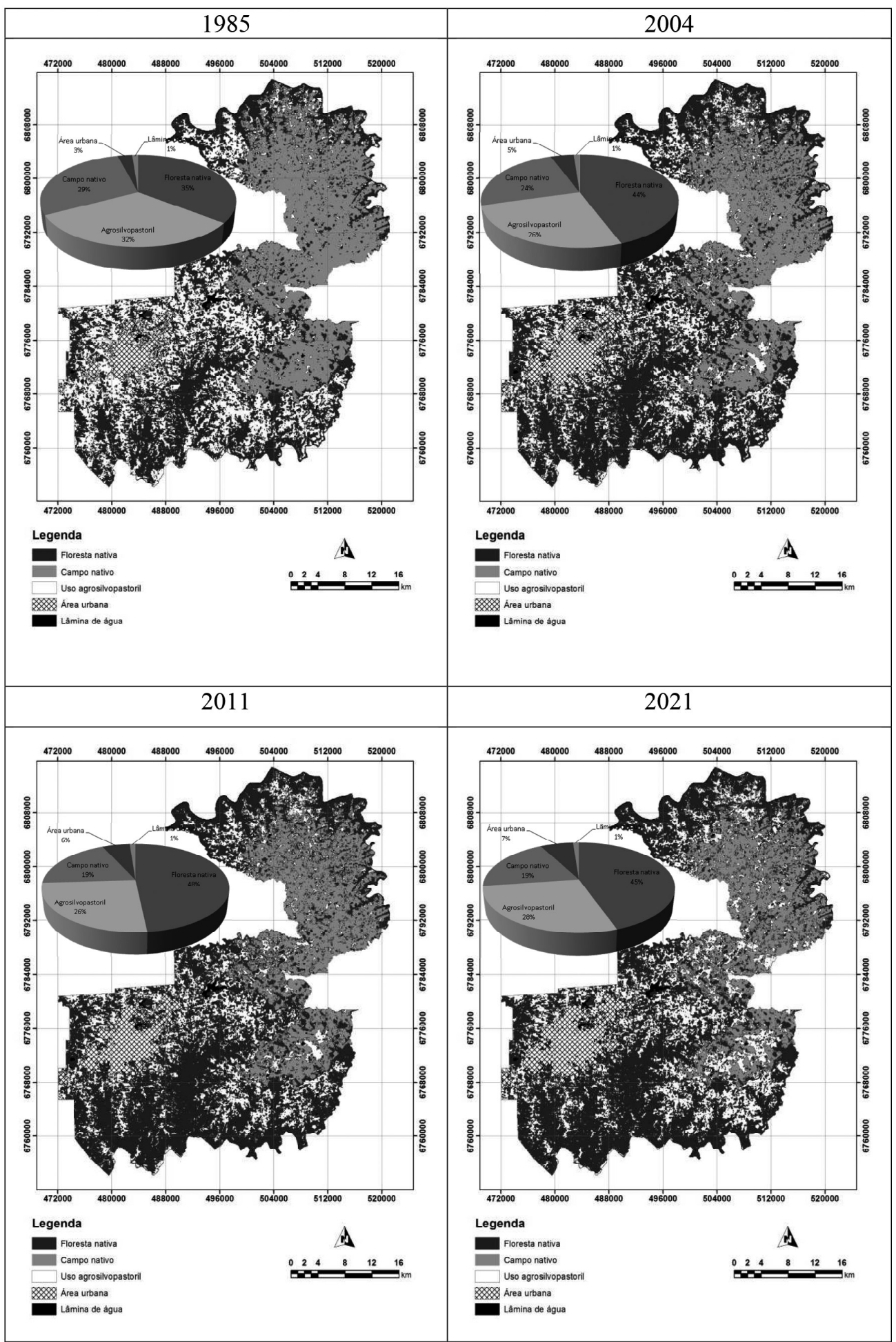

Figura 2 - Dados de uso e cobertura do solo.

Figure 2-Data use and land cover.

Revista Árvore, Viçosa-MG, v.39, n.6, p.1019-1030, 2015 
Na paisagem avaliada, evidenciou-se que de 1985 a 2011 houve aumento na ordem de $36 \%\left(203 \mathrm{~km}^{2}\right)$ das áreas que englobam a classe de floresta nativa, representado pela Floresta Estacional Decidual e Floresta Ombrófila Mista - Mata de Araucária. Esse aumento das áreas de floresta nativa está possivelmente relacionado com o êxodo rural e também com o abandono das terras de declividade mais acentuada, difíceis de ser cultivadas, por exigirem práticas conservacionistas intensivas no controle da erosão dos solos, na dificuldade do emprego de máquinas agrícolas e por apresentarem solos mais rasos e com grande quantidade de rochas particionadas.

Em contraponto, as áreas ocupadas pelos campos nativos (estepe gramíneo-lenhosa) tiveram redução considerável de, aproximadamente, $33,36 \%$ de sua área de 1985 para 2011. Observou-se o avanço da fruticultura, principalmente da maçã e uva, além da expansão do gado de corte, o que tem feito os produtores manejarem o campo com o plantio de pastagens, como aveia e azevém (no inverno) e milho (no verão).

Nas áreas de uso agrossilvipastoris estão incluídos os locais com o desenvolvimento da agricultura (incluindo o solo exposto), pastagens e estradas. Essa classe teve decréscimo entre os anos 1985 e 2011, passando de 488,68 km² para $357,35 \mathrm{~km}^{2}$, uma diminuição da ordem de $26 \%$. Os principais locais onde houve perda de área dessa classe são os de declividade mais acentuada, em que a utilização agrícola é dificultada devido à inviabilidade de aplicação da mecanização, por apresentar solos mais pobres em nutrientes e elevado grau de pedregosidade.

A área urbana também teve incremento considerável, passando de $46,02 \mathrm{~km}^{2}$ para $89,94 \mathrm{~km}^{2}$, uma variação de aproximadamente 95,44\%. Está, em termos territoriais, entre as maiores áreas urbanas do Estado e, em números populacionais, ocupa a segunda posição, com aproximadamente de 435 mil habitantes (IBGE, 2010). Essa grande variação está relacionada, sobretudo, com as características econômicas do município. Por ser considerado o principal polo metalmecânico do Estado do Rio Grande do Sul, Caxias do Sul apresenta taxa de empregabilidade elevada, atraindo trabalhadores de vários municípios do interior, inclusive de outros Estados, o que favorece esse crescimento acentuado observado nesses últimos 26 anos.

\subsection{Modelagem dinâmica espacial}

$\mathrm{Na}$ análise do cenário futuro, foram utilizados os dados de uso e cobertura do solo dos anos 1985, 2004 e 2011 e informações relativas ao relevo (hipsometria e clinografia), pedologia e distância dos fragmentos florestais, sendo esta última calculada e elaborada pelo próprio DINAMICA a partir do mapa de uso e cobertura do solo de entrada e recalculado a cada ano modelado. A paisagem simulada foi gerada anualmente, entre os períodos de 1985 a 2004, 2004 a 2011 e 2011 a 2021.

\subsubsection{Matrizes de transição anual e global}

A tabulação cruzada dos mapas de uso e cobertura do solo de 1985 e 2004 gerou a matriz de transição anual (unitária) e global (periódica), sendo a primeira utilizada no processo de modelagem (Tabela 1).

Esse dado tem por função quantificar as tendências da dinâmica da paisagem ao longo dos 19 anos analisados e mostrar quais as classes de uso e cobertura do solo permaneceram ou transacionaram para outras classes no período avaliado. Na diagonal principal, são indicados os valores de permanência de uma classe ao longo do tempo, enquanto os demais valores apontam a ocorrência de mudança entre as classes analisadas. A diagonal principal indica as permanências da classe, e o valor zero mostra a não ocorrência de modificação em uma classe para outra. Observou-se a transição de campo e de uso agrossilvipastoril para áreas florestais. Em alguns locais, as áreas de floresta foram convertidas para uso agrossilvipastoril e área urbana, principalmente naquelas áreas de relevo plano e situado nas proximidades do núcleo urbano observado em 1985.

\subsubsection{Pesos de evidência}

Os pesos de evidência representam a influência de uma variável na probabilidade espacial de uma transição de uma classe de uso e cobertura do solo para outra classe. Após a geração da matriz de transição foram calculados os pesos de evidência para as três variáveis estáticas (altitude, declividade e pedologia) e uma variável dinâmica (distância dos fragmentos florestais nativos a partir do ano 1985)

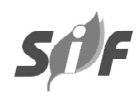

Revista Árvore, Viçosa-MG, v.39, n.6, p.1019-1030, 2015 
Tabela 1 - Matriz de transição anual decomposta entre os anos 1985 e 2004.

Table 1 - Matrix of annual transition decomposed between the years 1985 and 2004.

\begin{tabular}{lccccc}
\hline Classes & \multicolumn{4}{c}{ Classes } \\
\hline & Floresta nativa & Campo nativo & Agrossilvipastoril & Urbano & Água \\
Floresta nativa $^{1}$ & $\mathbf{0 , 9 9 9 6 9 7 3 7 2}$ & 0 & 0 & 0,000302627 & 0 \\
Campo nativo $^{3}$ & 0,001540230 & $\mathbf{0 , 9 8 2 4 6 3 8 7 8}$ & 0,008908547 & 0 & 0 \\
Agrossilvipastoril $^{3}$ & 0,014747542 & 0 & $\mathbf{0 , 9 8 2 4 6 3 8 7 8}$ & 0,002788579 & 0 \\
Urbano $^{4}$ & 0 & 0 & 0 & $\mathbf{1}$ & 0 \\
Água $^{5}$ & 0 & 0 & 0 & 0 & $\mathbf{1}$ \\
\hline
\end{tabular}

1. Mata nativa; 2. Estepe gramíneo-lenhosa; 3. Uso agrossilvipastoril; 4. Área urbana; e 5. Lâmina d'água.

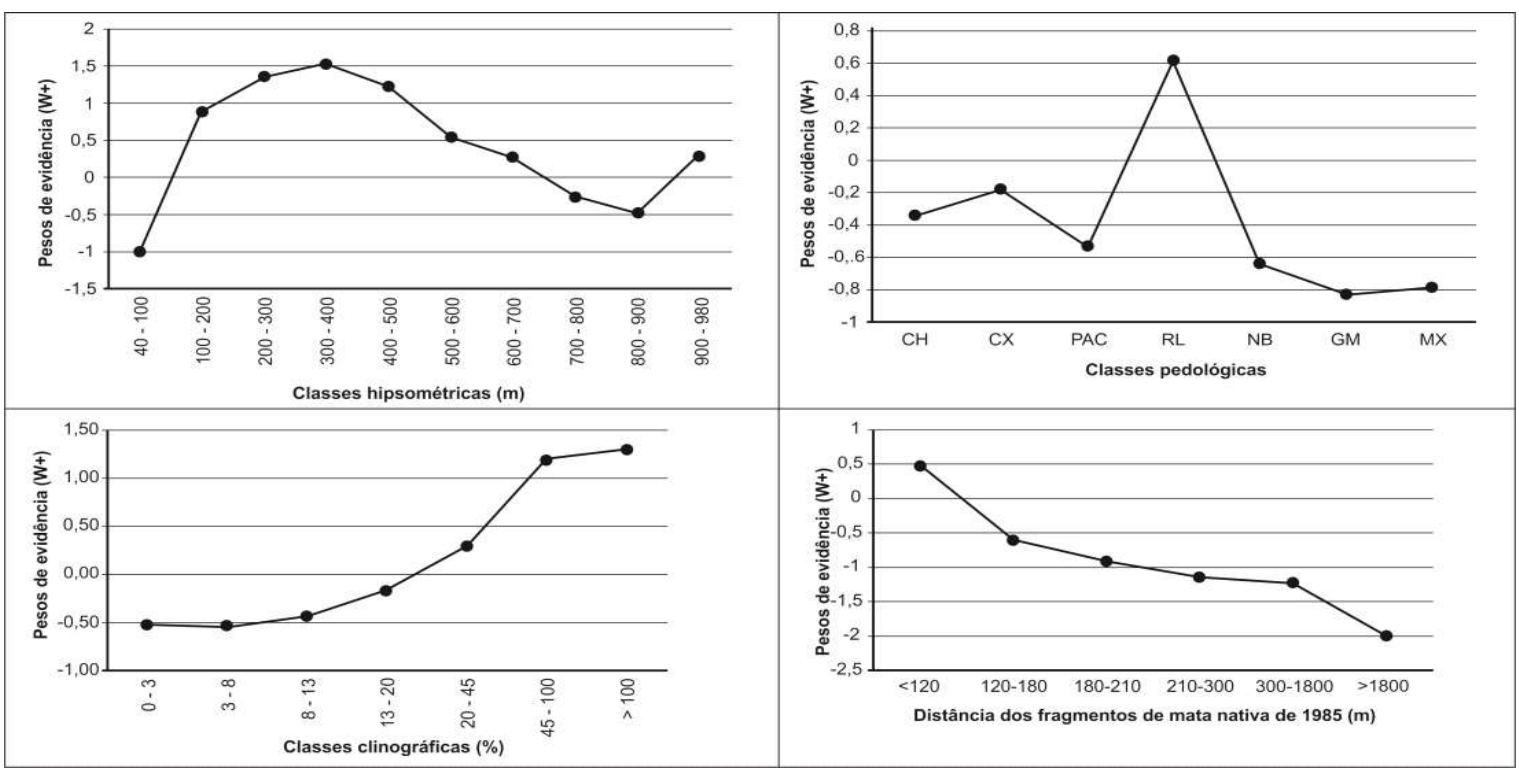

Figura 3 - Pesos de evidência das variáveis utilizadas na modelagem dinâmica espacial.

Figure 3 - Weights of evidence of the variables used in spatial dynamic modeling.

em relação à transição analisada, conforme mostrado na Figura 3. Quanto mais alto o valor do peso de evidência para cada classe da variável explicativa, maior é a probabilidade de as transições ocorrerem nesses locais.

Analisando os pesos de evidência apresentados na Figura 3 das quatro variáveis, verificou-se o comportamento da regeneração da floresta nativa em áreas que apresentavam usos agrossilvipastoris em 1985. De maneira geral, observou-se que a regeneração da floresta nativa ocorreu em declividades mais acentuadas (superiores a 45\%), em altitudes intermediárias (entre 200 e 500 m), em áreas de ocorrência de Neossolos litólicos e em distâncias de até $120 \mathrm{~m}$ dos fragmentos de mata consolidados em 1985 e dos anos subsequentes modelados.

\subsubsection{Calibração, simulação e validação do modelo de 1985 a 2004}

Após o cálculo dos pesos de evidência, os últimos parâmetros ajustados foram os percentuais de Expander e de Patcher, que necessitaram da indicação do tamanho médio dos fragmentos em hectares, da variância dos fragmentos em hectares e da forma dos fragmentos a serem simulados a cada iteração do modelo. Foram realizadas simulações com diferentes valores de Expander e Patcher, forma (isometria), saturação, tamanho médio e variância. Foram realizadas várias simulações com diferentes valores de Expander e Patcher. O melhor resultado foi obtido utilizando 0,5 de Expander e 0,5 de Patcher, 0,8 na forma dos fragmentos gerados por Expander, 0,4 na forma dos fragmentos gerados pelo Patcher, tamanho médio dos 
fragmentos igual a 5 ha e a variância dos fragmentos igual a 3 ha.

Os resultados das simulações foram validados segundo os procedimentos de Ajuste por Múltiplas Resoluções (F), descrito por Constanza (1989), que comparou o ajuste do mapa de uso e cobertura do solo simulado de 2004 com o mapa de uso e cobertura do solo observado para 2004, pela Medida de Similaridade Fuzzy (S), proposto por Hagen (2003). O melhor ajuste, tanto para $\mathrm{F}$ quanto para $\mathrm{S}$, foi nas janelas de varredura maiores, embora os valores do ajuste $F$ para todas as janelas de varredura fossem superiores a 0,9 e, para a similaridade $\mathrm{S}$, apenas para a janela de 1 x 1 , o valor ficou abaixo de 0,5 .

\subsubsection{Simulação e validação do modelo de 2004 a 2011}

Calibrado e validado o modelo para o período de 1985 a 2004, procedeu-se à simulação do cenário futuro para o ano 2011, a fim de realizar uma segunda validação a partir dos parâmetros utilizados para chegar ao cenário de 2004. Evidenciou-se que para os valores de F os resultados são similares aos encontrados para 1985 a 2004. Ao avaliar os valores de S, evidencia-se pequena diminuição na qualidade da simulação, principalmente para as janelas de varredura de menor tamanho ( $1 \mathrm{x}$ 1 e $2 \times 2$ ). Ressalta-se que o processo de mudança de uso do solo não segue um modelo linear, sendo influenciado por vários elementos, como as políticas de governo, elementos econômicos do período analisado, entre outros. Esse resultado era esperado, uma vez que, para modelar o cenário de 2011, foram utilizados os parâmetros calibrados para o período de 1985 a 2004. Mas, mesmo utilizando os parâmetros calibrados de 1985 a 2004, alcançou-se um cenário simulado muito próximo ao de 2011 , de modo que esses parâmetros são adequados para simular o cenário de 2021 .

\subsubsection{Simulação do modelo de 2011 a 2021}

Validado o modelo, procedeu-se à simulação do cenário para 2021. A Tabela 2 e a Figura 2 ilustram o resultado obtido pela modelagem dinâmica espacial.

Analisando o cenário da paisagem simulada para 2021, verificou-se a regeneração da mata nativa próxima dos fragmentos de mata, principalmente na paisagem do ano 2011, sendo um indicativo da expansão gradual de forma radial de indivíduos a partir da borda dos fragmentos florestais ou, também, por meio do estabelecimento de ilhas de vegetação, sobretudo na região dos campos. Observa-se na Tabela 2 aumento de 20,50\% das áreas de mata de 1985 a 2021 . Analisando esse dado apresentado na Figura 2, verificou-se maior agregação das áreas florestais, sendo um bom indicativo de qualidade ambiental.

\section{DISCUSS $\tilde{A} O$}

As imagens do satélite TM/Landsat 5 permitiram identificar e quantificar o aumento de aproximadamente $203 \mathrm{~km}^{2}$ das áreas florestais do bioma Mata Atlântica entre os anos 1985 e 2011, correspondendo a um incremento da ordem 36\% das Florestas Estacional Decidual e Ombrófila Mista. Périco et al. (2006), em uma região de Mata Atlântica, observou o avanço das áreas florestais entre os anos 1985 e 2002 de aproximadamente 30\%, corroborando, em termos de processos e dinâmica, o resultado encontrado na área de estudo. Em contrapartida, houve diminuição nas áreas dos campos de nativos (espete gramíneo-lenhosa) na ordem de 33\%. Esse resultado é confirmado pelo avanço dos plantios de Pinus elliotti, das atividades agrícolas, principalmente fruticultura (maça e uva) e o manejo do campo, com o plantio de gramíneas nativas e exóticas como alternativa de forrageio para o gado. Destaca-se que os campos

Tabela 2 - Uso e cobertura do solo de 1985 e do cenário simulado no ano 2021.

Table 2 - Use and land cover of 1985 and the simulated scenario in the year 2021.

\begin{tabular}{|c|c|c|c|c|c|c|}
\hline \multirow{3}{*}{ Classe } & \multicolumn{6}{|c|}{ Ano } \\
\hline & \multicolumn{2}{|c|}{1985} & \multicolumn{2}{|c|}{2021} & \multirow[b]{2}{*}{ Variação $\left(\mathrm{km}^{2}\right)$} & \multirow[b]{2}{*}{ Variação (\%) } \\
\hline & $\mathrm{km}^{2}$ & $\%$ & $\mathrm{~km}^{2}$ & $\%$ & & \\
\hline Floresta nativa & 563,77 & 35,52 & 707,51 & 44,58 & 143,74 & 20,50 \\
\hline Uso agrossilvipastoril & 501,87 & 31,62 & 446,33 & 28,12 & $-55,54$ & $-11,07$ \\
\hline Campo nativo & 456,43 & 28,76 & 309,02 & 19,47 & $-147,41$ & $-32,30$ \\
\hline Área urbana & 46,02 & 2,90 & 105,22 & 6,63 & $+59,20$ & 128,64 \\
\hline Lâmina d'água & 18,86 & 1,19 & 18,86 & - & - & - \\
\hline TOTAL & 1.587 & 100 & 1.587 & 100 & - & - \\
\hline
\end{tabular}


de altitude são protegidos pela Lei da Mata Atlântica - Lei Federal $n^{\circ} 11.428 / 2006$ (BRASIL, 2006). No entanto, somente em 2010 foram definidos os estágios sucessionais das vegetações primária e secundária desse ecossistema associado, com a publicação da Resolução do CONAMA $n^{\circ} 423 / 2010$ (BRASIL, 2010), permitindo identificar as formas permissíveis de manejo dessa vegetação, a partir desse enquadramento. Nesse aspecto, qualquer intervenção na região é passível de licenciamento ambiental, e o manejo em áreas compostas por vegetação primária e por vegetação em estágio avançado de regeneração somente será autorizado em caráter excepcional, quando necessários à realização de obras, projetos ou atividades de utilidade pública, pesquisas científicas e práticas preservacionistas. Na modelagem dinâmica espacial, evidenciou-se a regeneração das áreas de mata nativa de 1985 a 2021 em 20,50\%, principalmente nas encostas mais íngremes (declividade maior de $20 \%$ ), corroborando os dados obtidos por Silva et al. (2007), em locais de ocorrência de Neossolos litólicos e em altitudes variando entre 200 e $500 \mathrm{~m}$. Silva et al. (2012), em estudo conduzido em fragmentos de Mata Atlântica na região de transição entre a depressão central gaúcha e o Planalto Meridional, evidenciaram que as áreas com vegetação natural praticamente triplicaram. Hendges et al. (2012), avaliando a dinâmica das áreas de floresta nativa entre os anos 1988 e 2020, no Estado do Rio Grande do Sul, evidenciaram incremento na ordem de $32 \%$ das áreas de mata nativa. Além disso, verificou-se que essa regeneração ocorreu em áreas antes ocupadas por atividades antrópicas, seguidas pelos campos naturais.

\section{CONCLUSÃO}

Os dados de sensoriamento remoto permitiram obter um conjunto de informações temporais sobre o uso e cobertura do solo da área de estudo, o que possibilitou a calibração e validação da modelagem dinâmica espacial. Em conjunto com os dados referentes ao relevo e à pedologia, foi possível compreender quais variáveis mais contribuíram para a modificação da paisagem. Declividades mais acentuadas e com a ocorrência de Neossolos litólicos foram os locais onde se observou a maior regeneração da floresta nativa. Recomendase que, em trabalhos futuros, outras variáveis estáticas sejam incorporadas ao modelo, como a rede viária e os recursos hídricos. Esses dois elementos podem também explicar as razões pelas quais ocorre a dinâmica de mudança do uso e cobertura do solo.

\section{REFERÊNCIAS}

ACHARD, F.; EVA, H.D.; STIBIG, H.J.; MAYAUX, P.; GALLEGO, J.; RICHARDS, T.; MALINGREAU, J.P. Determination of deforestation rates of the world's humid tropical forests. Science, v.297, p.999-1002, 2002.

AGUIAR, A.P.D.; CÂMARA, G.; ESCADA, M.I.S. Spatial statistical analysis of land-use determinants in the Brazilian Amazonia: Exploring intra-regional heterogeneity. Ecological Modelling, v.209, n.1, p.169-188, 2007.

ALMEIDA, C.M.; MONTEIRO, A.M.V.; CÂMARA, G. Perspectiva histórica de modelos de dinâmicas urbanas e regionais. In: Geoinformação em urbanismo: cidade real x cidade virtual. São Paulo: Oficina de Textos, 2007. 368 p.

BRASIL. Casa Civil. Lei Federal no 11.428, de 22 de dezembro de 2006 . Dispõe sobre a utilização e proteção da vegetação nativa do Bioma Mata Atlântica, e dá outras providências. Disponível em: http://www.planalto.gov.br/ ccivil_03/_ato2004-2006/2006/lei/111428.htm. Acesso em: 10 abr. 2014.

BRASIL. Ministério da Agricultura. Resolução do Conselho Nacional de Meio Ambiente $n^{\circ}$ 423, de 12 de abril de 2010. Dispõe sobre parâmetros básicos para identificação e análise da vegetação primária e dos estágios sucessionais da vegetação secundária nos Campos de Altitude associados ou abrangidos pela Mata Atlântica. . Disponível em: < $\underline{\text { http:// }}$ www.mma.gov.br/port/conama/ legiabre.cfm?codlegi $=628>$. Acesso em: 15 set. 2014.

BRASIL. Ministério do Meio Ambiente.

Biodiversidade brasileira: avaliação e identificação de áreas e ações prioritárias para a conservação, utilização sustentável e repartição dos benefícios da biodiversidade nos biomas brasileiros. Brasília: 2002. 404 p.

BRASIL. Ministério do Meio Ambiente. Mapas de cobertura vegetal dos biomas brasileiros. Brasília: 2007. 16p.

CONSTANZA, R. Model goodness of fit: a multiple resolution procedure. Ecological Modelling, v.47, n.1.p.199-215, 1989. 
DEAN, W. A ferro e fogo: a história e a devastação da Mata Atlântica brasileira. São Paulo: Companhia das Letras, 1996. 484p.

FAO. Food and Agriculture Organization of the United Nations. The state of the world's forests. Roma: Electronic Publishing Policy and Support Branch. Communication Division; 2007. $144 \mathrm{p}$.

FLAMENCO-SANDOUAL, A.; MARTINEZ RAMOS, M.; MASERA, O.R. Assessing implications of land-use and land-cover change dynamics for conservation of a highly diverse tropical rain forest. Biological

Conservation. v.138, n.1, p.131-145, 2007.

FLORES, C. A. F. (Org.). Levantamento semidetalhado de solos: Região da Serra Gaúcha-RS. Porto Alegre: UFRGS - Centro de Ecologia, 2007.

FONSECA, G.A.B. The vanishing Brazilian Atlantic forest. Biological Conservation, v.34, n.1, p.17-34, 1985.

GLCF. Global Land Cover Facility. GeoCover Technical Guide. Maryland: University of Institute for Advanced Computer Studies, 2008.

GOODCHILD, M.F. Geographical data modeling. Computers \& Geosciences, v.4, n.18, p.401-408, 1992.

HAGEN, A. Fuzzy set approach to assessing similarity of categorical maps. Int. Journal of Geographical Information Science, v.17, n.3, p.235-249, 2003.

HASENACK, H. Base cartográfica vetorial contínua do Rio Grande do Sul na escala 1:50.000. Porto Alegre: UFRGS, Centro de Ecologia, 2010.

HENDGES, E.R.; PEREIRA, R.S.; ANDRES, J. Dinâmica das áreas de floresta nativa no Rio Grande do Sul no período de 1988 a

2020. Ciência Rural, v.42, n.5, p.828-833, 2012.

HOBBS, R.J. Markov models in the study of postfire succession in healthland communities.

Vegetation, v.56, n.1, p.17-30, 1983.
INSTITUTO BRASILEIRO DEGEOGRAFIA E ESTATÍSTICA - IBGE. Pesquisa Nacional por Amostra de Domicílios. Síntese dos Indicadores de 2009. Rio de Janeiro: 2010.

INSTITUTO NACIONAL DE PESQUISAS ESPACIAIS - INPE. Projeto PRODES: monitoramento do desmatamento das formações florestais na Amazônia Legal. Disponível em: http:// www.obt.inpe.br/prodes/index.php. Acesso em: 01 maio. 2015.

LEWINSOHN, T. M. (Coord.). Avaliação do estado do conhecimento da biodiversidade brasileira. Brasília, D.F.: Ministério da Agricultura, 2005. v. 2.

LIMA, T.C.; GUILLEN-LIMA, C.M.; OLIVEIRA, M.S.; SOARES-FILHO, B.S. DINAMICA EGO e Land Change Modeler para simulação de desmatamento na Amazônia brasileira: análise comparativa. In: Simpósio Brasileiro de Sensoriamento Remoto, 16. 2013, São José dos Campos. Anais... São José dos Campos: INPE, 2013. p.6379-6386.

MAEDA, E.E.;ALMEIDA, C.M.; XIMENES, A.C.; FORMAGGIO,A.R.;SHIMABUKURO, Y.E.; PELLIKKA, P. Dynamic modeling of forest conversion: simulation of past and future scenarios of rural activities expansion in the fringes of the Xingu National Park, Brazilian Amazon.

International Journal of Applied Earth Observation and Geoinformation. v.13, n.3, p.435-446, 2011.

MESQUITA, C.A.B.; HOLVORCEM, C.G.D.A.; TAMBOSI, L.R.; SILVA, S.C.. Mosaicos florestais sustentáveis: Monitoramento integrado da biodiversidade e diretrizes para restauração florestal. Rio de Janeiro: Instituto BioAtlântica, 2011.

METZGER, J.P.; MARTENSEN, A.C.; DIXO, M.; BEMACCI, L.C.; RIBEIRO, M.C.; TEIXEIRA, A.M.G.; PARDINI. R.. Time-lag in biological responses to landscape changes in a highly dynamic Atlantic forest region. Biology Conservation, v.142, p. $1166-1177,2009$.

MITTERMEIER, R.A.; GIL, P.R.; HOFFMANN, M.; PILGRIM, J.; BROOKS, T.; MITTERMEIER, C.G.; LAMOUREX, J.; FONSECA, G.A.B. Hot spots revisited. Mexico City: CEMEX, 2004. 392p.

Revista Árvore, Viçosa-MG, v.39, n.6, p.1019-1030, 2015 
MYERS, N. Tropical deforestation and remote sensing. Forest Ecology and Management, v.23, p.215-225, 1988.

MYERS, N.; MITTERMEIER, R.; MITTERMEIER, C.; FONSECA, G.; KENT. J. Biodiversity hotspots for conservation priorities. Nature. v.403, p.853858,2000 .

PEDROSA, B. M.; CÂMARA, G. Modelagem dinâmica: conceitos básicos e exemplos de sistemas. In: ALMEIDA, C.M.; MONTEIRO, A.M.V.; CÂMARA, G. Geoinformação em urbanismo: cidade real $x$ cidade virtual. São Paulo: Oficina de Textos, 2007. p.86-105.

PÉRICO, E.; CEMIN, G.; REMPEL, C. Caracterização da paisagem do município de Arvorezinha, RS, com ênfase na dinâmica dos fragmentos florestais, por meio de sistemas de informações geográficas (SIGs). Scientia Forestalis, n. 70, p. 09-21, 2006.

PIONTEKOWSKI, V. J.; SILVA, S. S.; MENDONZA, E. R. H.; COSTA, W. L. S.; RIBEIRO, F. C. Modelagem do desmatamento para o Estado do Acre utilizando o programa Dinamica EGO. In: SIMPÓSIO DE GEOTECNOLOGIAS NO PANTANAL, 4., 2012, Bonito, MS.

Anais...Brasília, DF: Embrapa, 2012. p.1064-1075.

SILVA, J.F.; ECKHARDT, R.R.; REMPEL, C.

Análise temporal e modelagem ambiental da cobertura florestal nativa do município de Roca Sales-RS. Revista Brasileira de

Cartografia, n.64,n.4, p.629-642, 2011

SILVA, W.G.; METZGER, J.P.; SIMOES, S.; SIMONETTI, C. Relief influence on the spatial distribution of the Atlantic Forest cover on the
Ibiúna Plateau, SP. Brazilian Journal Biology, v.67, n.3, p.403-441, 2007.

SOARES-FILHO, B.S. Modelagem da dinâmica de paisagem de uma região de fronteira de colonização amazônica. 1998. Tese (Doutorado Engenharia de Transportes) - Universidade de São Paulo, São Paulo, 1998.

SOARES-FILHO, B.S.; ALENCAR, A.; NEPSTAD, D.; CERQUEIRA, G.; VERA DIAZ, M.; RIVERO, S.; SOLORZANO, L.; VOLL, E. Simulating the response of land-cover changes to road paving and governance along a major Amazon highway: the Santarém-Cuiabá corridor. Global Change Biology, v. 10, p.745-764, 2004.

SOARES-FILHO, B.S.; GARCIA, R.A.; RODRIGUES, H.O.; MORO, S.; NEPSTAD, D. Coupling socioeconomic and demographic dimensions to a spatial simulation model of deforestation for the Brazilian Amazon. In: SCIENCE TEAM MEETING IN SALVADOR, 11 ., 2007, Salvador. Presentation...Salvador: 2007. Abstract, 60

SOARES-FILHO, B.S.; NEPSTAD, D.C.; CURRAN, L.M.; CERQUEIRA, G.C.; GARCIA, R.A.; RAMOS C.A.; VOLL, A.; MCDONALD, A.; LEFEBVRE, P.; SCHLESINGER, P. Modelling conservation in the Amazon basin. Nature, v.440, p.520-523, 2006.

TEIXEIRA, A.M.G.; PARDINI. R.. Time-lag in biological responses to landscape changes in a highly dynamic Atlantic forest region. Biology Conservation, v.142, p. 1166-1177, 2009.

TEIXEIRA, M.B.; NETO, A.B.C. Vegetação. In: Projeto RADAM BRASIL. Folha SH. 22 Porto Alegre e parte da Folha SI. 22 Lagoa Mirim. Rio de Janeiro: IBGE, 1986. 